\title{
Prevalence and characteristics of polycystic ovarian syndrome in a sample of infertile Kurdish women attending IVF infertility center in maternity teaching hospital of Erbil City
}

\author{
Bayan Hussein, Shahla Alalaf* \\ Department of Obstetrics and Gynaecology, College of Medicine, Hawler Medical University, Erbil City, Iraq \\ Email: "shahla_alaf@yahoo.com
}

Received 29 July 2013; revised 26 August 2013; accepted 2 September 2013

Copyright (C) 2013 Bayan Hussein, Shahla Alalaf. This is an open access article distributed under the Creative Commons Attribution License, which permits unrestricted use, distribution, and reproduction in any medium, provided the original work is properly cited.

\begin{abstract}
Objective: To estimate the prevalence of polycystic ovarian syndrome (PCOS), and to compare the clinical, hormonal and ultrasonography features between infertile women with or without PCOS. Design: A descriptive, comparative study. Materials and Methods: This study was conducted from May 1, 2007 to August 1, 2008, in the Infertility Care and IVF center in Maternity Teaching Hospital, Erbil city, Kurdistan region, North of Iraq. A total of 320 infertile women aged 18 - 45 years, were evaluated for clinical features (oligo-/amenorrhea, hirsutism), body mass index, waist-hip ratio (WHR) and hormonal measures. Transvaginal ultrasonography was used to assess the ovarian morphology. The Rotterdam 2003 criteria adopted by the European Society for Human reproduction and Embryology and the American Society for Reproductive Medicine were used to diagnose cases of PCOS. Data analysis was performed using the SPSS version 15. Results: The prevalence of PCOS was $33 \%$. There were significant differences between the two groups in terms of oligo-/amenorrhea, hirsutism, WHR, and ovarian ultrasound features. There were no significant differences between the two groups in correlations between the level of obesity with the incidences of anovulation, hyperandrogenemia and hirsutism or with hormonal features. Conclusions: A high prevalence rate of PCOS was observed among infertile women attending IVF center using the Rotterdam 2003 criteria for diagnosis.
\end{abstract}

Keywords: Amenorrhea; Hirsutism; Oligomenorrhea;

"Corresponding author.
Polycystic Ovaries; Polycystic Ovarian Syndrome

\section{INTRODUCTION}

Polycystic ovarian syndrome (PCOS) is the most common gynecological endocrinopathy [1]. PCOS appears to be associated with an increased risk of metabolic aberrations, including insulin resistance and hyperinsulinism, type 2 diabetes mellitus, dyslipidemia, cardiovascular disease, and endometrial carcinoma [2].

It is commonly diagnosed in young women with anovulatory infertility, oligomenorrhea or hyperandogenic problems such as hirsutism and acne. Although associated with obesity, the syndrome is also frequently seen in women of normal body conformation [3].

Although the exact definition of PCOS has varied when described by various experts, following a Consensus conference held in Rotterdam in 2003; an internationally accepted definition has been adopted by the European Society for Human Reproduction and Embryology and American Society for Reproductive Medicine, the ESHRE/ASRM Rotterdam consensus (Rotterdam consensus) [4]. According to this consensus, two of the following three diagnostic criteria must be met to arrive at a proper diagnosis:

1) Oligo-ovulation or anovulation;

2) Hyperandrogenemia/hyperandrogenism and/or;

3) Polycystic ovaries.

Estimations of the prevalence of PCOS depend on the population being assessed, as there are ethnic differences in the clinical and biochemical features of PCOS [5].

The reported prevalence of PCOS ranges between $2.2 \%$ to $26 \%$ in various countries, depending on the recruitment method, the study population, the criteria used for its definition and the method used to define each cri- 
terion [6]. The prevalence of PCOS can be as high as $30 \%$ in women with secondary amenorrhea, $40 \%$ in women with infertility, $75 \%$ in women with oligomenorrhea and $90 \%$ in women with hirsutism [7].

This study is regarded the first to be conducted in our city to determine the prevalence and characteristics of women with PCOS using the ESHRE/ASRM Rotterdam consensus criteria in a sample of infertile Kurdish population.

\section{OBJECTIVES}

The primary objectives were:

- To estimate the prevalence of PCOS among a sample of infertile Kurdish women attending the Infertility Care and IVF center in our teaching hospital; and

- To analyze the diagnostic criteria for PCOS in this context.

The secondary objective was to evaluate and compare the demographic characteristics, clinical, biochemical and ultrasonography features of infertile women with and without PCOS.

\section{MATERIALS AND METHODS}

\subsection{Subjects and Setting}

This study was carried out on infertile women attending the Infertility Care and IVF Center in Erbil City, North Iraq, Kurdistan region. It is the single infertility center in the city and more than 3200 infertile couples have been treated. From May 1, 2007 to August 1, 2008 all infertile women attending the center seeking gynecological advice were included in the study. Women with other causes of endocrinological abnormalities such as primary hyperprolactinemia, thyroid dysfunction, Cushing's syndrome and congenital adrenal hyperplasia were excluded from the PCOS study group.

\subsection{Definitions}

The diagnostic criteria used to identify the presence of PCOS were according to the criteria proposed at the Rotterdam revised consensus meeting [8]. Amenorrhea was defined as an absence of menstrual cycles for more than 6 months. Oligomenorrhea was defined as a delay in menses of $>35$ days to 6 months [9]. Clinical hyperandrogenism was diagnosed using a modified FerrimanGallwey score for evaluating and quantifying hirsutism in women using nine body areas (upper lip, chin, chest, upper and lower abdomen, thighs, upper and lower back and upper arm) [10]. Hair growth was rated from 0 (no growth of terminal hair) to 4 (extensive hair growth) in each of the nine locations; a score of 8 or higher was regarded as indicative of androgen excess. The presence of acne, greasy skin and thinning of hair of the scalp were also recorded, although no specific scoring systems were applied [11].

\subsection{Hyperandrogenemia}

This was defined as increased circulating levels of androgen. The normal range of total testosterone for women is $15-70(\mathrm{ng} / \mathrm{dl})$. A total testosterone level $>70$ $\mathrm{ng} / \mathrm{dl}$ was regarded as indicating hyperandrogenemia.

\subsection{Overweight and Obesity}

These parameters were defined according to WHO criteria as body mass index (BMI), calculated by dividing the weight in kilograms by height in meters squared. Being overweight was defined as a BMI $>25 \mathrm{~kg} / \mathrm{m}^{2}$ and being obese as $>30 \mathrm{~kg} / \mathrm{m}^{2}$

\subsection{Waist-to-Hip Ratio (WHR)}

This was calculated after measuring the waist circumference between pelvic brim and costal margin, while hip circumference was taken at the level of greater trochantor. A WHR $<0.85$ was considered normal, while a WHR $\geq 0.85$ was regarded abnormal [12].

\subsection{Ultrasonography}

Transvaginal ultrasonic screening with an $8 \mathrm{MHz}$ vaginal with $8 \mathrm{MHz}$ vaginal transducer (PHILIPS, Model 2540, USA), was performed twice for all 320 women. The first examination was performed in the early follicular phase during ovarian quiescence for evaluating ovarian morphology. Polycystic ovaries (PCOs) were diagnosed in the case of an increased follicular count ( $>11$ follicles, 2 $-9 \mathrm{~mm}$ in one or both ovaries) and/or an increased ovarian volume (>10 ml) for at least one ovary [13]. The second transvaginal ultrasound examination was done at mid-cycle for detecting mature follicles in cycles without the prior use of ovulation-inducing drugs. Follicles $>16$ $\mathrm{mm}$ in diameter were regarded as mature.

\subsection{Hormonal Analyses}

Serum samples were analyzed in non stimulated tracking cycles and the following hormonal investigations were performed on all women:

Days 2-3 of the menstrual cycle: follicle stimulating hormone (FSH) and luteinizing hormone (LH) levels; Total testosterone and prolactin levels; Day 21 progesterone level.

The normal cutoff for FSH and LH was taken as $<10$ $\mathrm{mIU} / \mathrm{ml}$. A prolactin level $<35 \mathrm{ng} / \mathrm{ml}$ was taken as nor$\mathrm{mal}$; a total testosterone level of $<70 \mathrm{ng} / \mathrm{dl}$ was regarded as normal and a progesterone level of $>20 \mathrm{ng} / \mathrm{ml}$ on day 21 was regarded as confirming ovulation. 


\subsection{Grouping of the Cases}

The presence of PCOS features was defined using the Rotterdam (2003) criteria. The 320 women were divided into two groups: a PCOS group and a non-PCOS group.

\subsection{Phenotypes}

Women considered to exhibit PCOS were classified into four phenotypes:

- Phenotype A: ovulatory dysfunction + hirsutism and/ or hyperandrogenism + PCOs (full blown syndrome);

- Phenotype B: ovulatory dysfunction + hirsutism and/ or hyperandrogenism;

- Phenotype C: ovulatory dysfunction + PCOs;

- Phenotype D: hirsutism and/or hyperandrogenism + PCOs $[14,15]$.

\subsection{Study Protocol}

A specially designed data collection form was prepared for recording information about each patient. This included a medical history, examination results, hormonal levels and ultrasonography findings. Patients were interviewed directly by one of the researchers.

\subsection{Ethical Approval}

The institutional Review Committee of Hawler Medical University, College of Medicine approved the study proposal (approval number: 17/N Date: 3/2/2008). An informed verbal consent was obtained from all participants.

\subsection{Statistical Analysis}

Data analysis was performed using IBM SPSS Statistics (version 15: IBM Corp., Armonk, NJ, USA). All data are reported as the means $\pm \mathrm{SD}$, Student t-test and Chi squared tests were used when appropriate. $P<0.05$ was considered statistically significant.

\section{RESULTS}

There were 106 women in the PCOS group and 214 in the non-PCOS group. The prevalence of PCOS within the study population was $33 \%$. Hyperprolactinaemia was detected in six women, and hyperthyroidism was detected in four; these women were included in the nonPCOS group.

The frequencies of PCOS phenotypes (Figure 1) were as follows:

-Phenotype A: $42 \%$;

-Phenotype B: $24 \%$;

-Phenotype C: $20 \%$;

-phenotype D: $14 \%$;

-Phenotype C: $20 \%$ of women with PCOS;

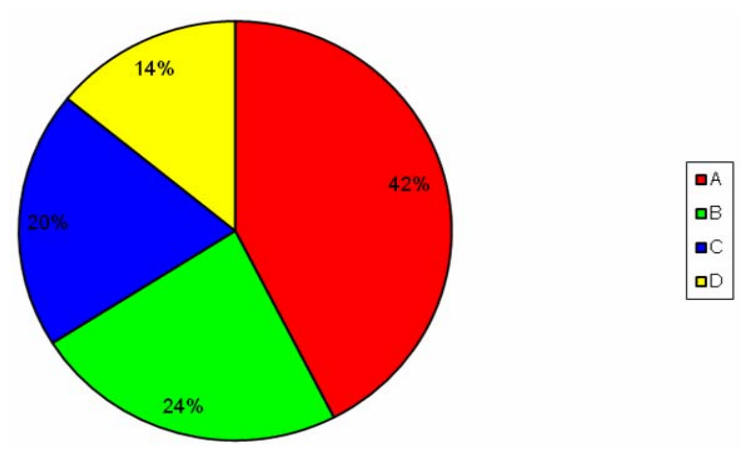

Figure 1. Distribution of women with PCOS according to phenotype.

-Phenotype D: consisting of $14 \%$ of women with PCOS.

The mean ages in the PCOS and non-PCOS groups were $29.5 \pm 5.45$ years versus $32.9 \pm 6.95$ years, respectively $(P=0.00061)$. Most women in the PCOS group $(30.2 \%)$ were aged $25-29$ years, while in the non-PCOS group most (24.3\%) were aged 35 - 39 years . There were no statistically significant difference between the 2 groups regarding educational level, occupation or residency. There were also no significant difference between the two groups regarding any family history of infertility and obesity (Table 1).

The mean age at marriage was not significantly less in the PCOS group than in the non-PCOS group. There was primary infertility in $61.3 \%$ of women in the PCOS group versus $58.4 \%$ in the non-PCOS group; the incidences of secondary infertility were $38.7 \%$ versus $41.6 \%$ respectively; these were not significantly different. Also the duration of infertility was not less in the PCOS group than in the non-PCOS group, as listed in Table 2.

Oligomenorrhea and amenorrhea were significantly more common among the PCOS women at $74.5 \%$ and $11.3 \%$, respectively, while in non-PCOS group the rate were $23.4 \%$ and $2.3 \%$ respectively $(P=0.000)$.

Women in the PCOS group had more coarse hair growth (hirsutism) than women in the non-PCOS group $(P=0.000)$.

Acne was not significantly more prevalent among women with PCOS than in women without PCOS: $22.6 \%$ versus $18.7 \%$ respectively $(P=0.405)$. There was a statistically significant difference between the two groups in reporting greasy skin and history of scalp hair loss. Women with PCOS had a higher mean BMI than women in the non-PCOS group but the difference was not significant statistically $\left(30.9 \pm 5.84 \mathrm{~kg} / \mathrm{m}^{2}\right.$ versus $29.9 \pm 5.31$ $\mathrm{kg} / \mathrm{m}^{2}$ respectively, $P=0.571$ ). There was no significant difference between the two groups in the incidence of obesity and being overweight. Mean WHR was significantly greater in the PCOS group $(0.96 \pm 0.06$ versus 0.92 $\pm 0.06 ; P=0.019$ ) (Table 3 ).

The incidence of elevated LH level was significantly higher in PCOS group than in non-PCOS group $(17 \%$ 
Table 1. Distribution of women according to demographic factors and family history of infertility and obesity.

\begin{tabular}{|c|c|c|c|c|c|}
\hline Characteristics & \multicolumn{2}{|c|}{ PCOS group } & \multicolumn{2}{|c|}{ Non-PCOS group } & $P$ \\
\hline \multirow[t]{2}{*}{ Mean age (years) } & \multicolumn{2}{|c|}{$29.5 \pm 5.454$} & \multicolumn{2}{|c|}{$32.9 \pm 6.957$} & 0.00061 \\
\hline & No. & $\%$ & No. & $\%$ & \\
\hline Residence & & & & & 0.476 \\
\hline Inside Erbil City & 61 & 57.5 & 132 & 61.7 & \\
\hline Outside Erbil City & 45 & 42.5 & 82 & 38.3 & \\
\hline Education & & & & & 0.922 \\
\hline College & 5 & 4.7 & 4 & 1.9 & \\
\hline Institution & 4 & 3.8 & 15 & 7.0 & \\
\hline Secondary school & 20 & 18.9 & 39 & 18.2 & \\
\hline Primary school & 47 & 44.3 & 88 & 41.1 & \\
\hline Illiterate & 30 & 28.3 & 68 & 31.8 & \\
\hline Occupation & & & & & 0.799 \\
\hline Employed & 17 & 16.0 & 32 & 15.0 & \\
\hline House wife & 89 & 84.0 & 182 & 85.0 & \\
\hline Family history & & & & & 0.165 \\
\hline No infertility or obesity & 27 & 25.5 & 64 & 29.9 & \\
\hline Infertility only & 40 & 37.7 & 67 & 31.3 & \\
\hline Obesity only & 27 & 25.5 & 70 & 32.7 & \\
\hline Infertility and obesity & 12 & 11.3 & 13 & 6.1 & \\
\hline Total & 106 & 100 & 214 & 100 & \\
\hline
\end{tabular}

Table 2. Distribution of women according to age at marriage, duration of infertility and type of infertility.

\begin{tabular}{|c|c|c|c|c|c|}
\hline \multirow{2}{*}{ Features } & \multicolumn{2}{|c|}{ PCOS group } & \multicolumn{2}{|c|}{ Non-PCOS group } & \multirow{2}{*}{$P$ value } \\
\hline & No. & $\%$ & No. & $\%$ & \\
\hline Age at marriage & & & & & 0.108 \\
\hline$<30$ years & 92 & 86.7 & 170 & 79.5 & \\
\hline$\geq 30$ years & 14 & 13.3 & 44 & 20.5 & \\
\hline Duration of infertility & & & & & 0.062 \\
\hline$\leq 2$ years & 17 & 16.0 & 54 & 25.3 & \\
\hline$<2$ years & 89 & 83.9 & 160 & 74.7 & \\
\hline Types of infertility & & & & & 0.617 \\
\hline Primary & 65 & 61.3 & 125 & 58.4 & \\
\hline Secondary & 41 & 38.7 & 89 & 41.6 & \\
\hline Total & 106 & 100 & 214 & 100 & \\
\hline
\end{tabular}


Table 3. Distribution of women in the PCOS and Non-PCOS groups according to clinical features.

\begin{tabular}{|c|c|c|c|c|c|}
\hline \multirow{2}{*}{ Clinical features } & \multicolumn{2}{|c|}{ PCOS group } & \multicolumn{2}{|c|}{ Non-PCOS group } & \multirow{2}{*}{$\boldsymbol{P}$} \\
\hline & No. & $\%$ & No. & $\%$ & \\
\hline Menstruation & & & & & 0.000 \\
\hline Amenorrhea & 12 & 11.3 & 5 & 2.3 & \\
\hline Oligomenorrhea & 79 & 74.5 & 50 & 23.3 & \\
\hline Regular & 15 & 14.1 & 159 & 74.3 & \\
\hline Hirsutism & & & & & 0.000 \\
\hline Positive & 83 & 78.3 & 80 & 37.4 & \\
\hline Negative & 23 & 21.7 & 134 & 62.6 & \\
\hline Acne & & & & & 0.405 \\
\hline Positive & 24 & 22.6 & 40 & 18.7 & \\
\hline Negative & 82 & 77.4 & 174 & 81.3 & \\
\hline \multicolumn{6}{|l|}{ Greasy skin } \\
\hline Positive & 68 & 64.2 & 104 & 48.6 & \\
\hline Negative & 38 & 35.8 & 110 & 51.4 & \\
\hline Scalp hair loss & & & & & 0.021 \\
\hline Positive & 60 & 56.6 & 92 & 43.0 & \\
\hline Negative & 46 & 43.4 & 122 & 57.0 & \\
\hline BMI (kg/m²) & & & & & 0.571 \\
\hline$<18$ & 0 & & 3 & 1.4 & \\
\hline $18-24.9$ & 55 & 51.9 & 111 & 51.9 & \\
\hline $25-30$ & 14 & 13.2 & 33 & 15.4 & \\
\hline$>30$ & 37 & 34.9 & 67 & 31.3 & \\
\hline WHR & & & & & 0.019 \\
\hline$\leq 0.85$ & 7 & 6.6 & 34 & 15.9 & \\
\hline$>0.85$ & 99 & 93.4 & 180 & 84.1 & \\
\hline Total & 106 & 100 & 214 & 100 & \\
\hline
\end{tabular}

versus $8.4 \%$ respectively, $P=0.000$ ). The incidence of elevated FSH level was not significantly higher in the non-PCOS group than in the PCOS group $(15 \%$ versus $10.4 \%$ respectively $=0.476$ ). There was no statistically significant difference between the two groups in terms of the LH/FSH ratio and total testosterone level. Progesterone was measured in the mid-luteal phase to confirm ovulation and there was a statistically significant difference between the two groups; $90.7 \%$ of the non-PCOS group versus $7.5 \%$ of PCOS group showed ovulatory cycles (Table 4).

Table 5 shows the ultrasonic appearance of polycystic ovaries in both groups.
Correlations between BMI and oligomenorrhea and WHR and oligomenorrhea in women with PCOS are shown in Tables 6 and 7. Oligomenorrhea increased with increasing BMI and WHR, but statistically these were not significant.

There were no significant correlations between BMI and hirsutism or WHR and hirsutism in women with PCOS. Likewise, there were no significant correlations between BMI and the mean LH/FSH ratio, and WHR and the mean LH/FSH ratio in women with PCOS. When comparing the BMI and WHR values with serum testosterone level in women with PCOS, the serum testosterone level was raised with increasing BMI and WHR but the 
Table 4. Distribution of hormone levels.

\begin{tabular}{|c|c|c|c|c|c|c|}
\hline \multirow{2}{*}{ Hormone Level } & \multicolumn{2}{|c|}{ PCOS group } & \multicolumn{2}{|c|}{ Non-PCOS group } & \multirow{2}{*}{ Total } & \multirow{2}{*}{$\boldsymbol{P}$} \\
\hline & No. & $\%$ & No. & $\%$ & & \\
\hline LH level & & & & & & 0.000 \\
\hline High & 18 & 17 & 18 & 8.4 & 36 & \\
\hline Normal & 88 & 83 & 196 & 91.6 & 284 & \\
\hline FSH level & & & & & & 0.476 \\
\hline High & 11 & 10.4 & 32 & 15 & 43 & \\
\hline Normal & 95 & 89.6 & 182 & 85 & 277 & \\
\hline LH/FSH ratio & & & & & & 0.140 \\
\hline$<2$ & 94 & 88.7 & 200 & 93.5 & 294 & \\
\hline$\geq 2$ & 12 & 11.3 & 14 & 6.5 & 26 & \\
\hline Testosterone level & & & & & & 0.088 \\
\hline High & 8 & 7.5 & 7 & 3.3 & 15 & \\
\hline Normal & 98 & 92.4 & 207 & 96.7 & 305 & \\
\hline Progesterone level & & & & & & 0.05 \\
\hline Low & 98 & 92.5 & 20 & 9.3 & 118 & \\
\hline High & 8 & 7.5 & 194 & 90.7 & 202 & \\
\hline Total & 106 & 100 & 214 & 100 & & \\
\hline
\end{tabular}

Table 5. Distribution of sample size according to transvaginal (US) findings.

\begin{tabular}{|c|c|c|c|c|c|}
\hline \multirow{2}{*}{ Transvaginal US finding } & \multicolumn{2}{|c|}{ PCOS } & \multicolumn{2}{|c|}{ Non-PCOS } & \multirow{2}{*}{$\boldsymbol{P}$} \\
\hline & No. & $\%$ & No. & $\%$ & \\
\hline PCSs features & & & & & 0.000 \\
\hline Yes & 81 & 76.4 & 56 & 26.2 & \\
\hline No & 25 & 23.6 & 158 & 73.8 & \\
\hline Mature follicle & & & & & 0.026 \\
\hline Yes & 14 & 13.2 & 51 & 23.8 & \\
\hline No & 92 & 86.8 & 163 & 76.2 & \\
\hline Total & 106 & 100 & 214 & 100 & \\
\hline
\end{tabular}

Table 6. Correlations between BMI and clinical and biochemical features of PCOS.

\begin{tabular}{|c|c|c|c|c|c|}
\hline PCOS features & $18-24.9 \mathrm{~kg} / \mathrm{m}^{2}$ & $25-30 \mathrm{~kg} / \mathrm{m}^{2}$ & $>30 \mathrm{~kg} / \mathrm{m}^{2}$ & Total & $P$ \\
\hline Menstruation & & & & & 0.553 \\
\hline Amenorrhea & 3 & 4 & 5 & 12 & \\
\hline Oligomenorrhea & 9 & 26 & 44 & 79 & \\
\hline Regular & 2 & 7 & 6 & 15 & \\
\hline Hirsutism & & & & & 0.403 \\
\hline Positive & 11 & 29 & 43 & 83 & \\
\hline Negative & 3 & 8 & 12 & 23 & \\
\hline \multicolumn{6}{|l|}{ LH/FSH ratio } \\
\hline$<2$ & 11 & 34 & 49 & 94 & \\
\hline$\geq 2$ & 3 & 3 & 6 & 12 & \\
\hline Testosterone level & & & & & 0.320 \\
\hline High & 7 & 2 & 6 & 8 & \\
\hline Normal & 14 & 35 & 49 & 98 & \\
\hline
\end{tabular}


Table 7. Correlations between WHR and clinical and biochemical features of PCOS.

\begin{tabular}{|c|c|c|c|c|}
\hline PCOS features & WHR $\leq 0.85$ & WHR $>0.85$ & Total & $P$ \\
\hline Menstruation & & & & 0.966 \\
\hline Amenorrhea & 11 & 1 & 12 & \\
\hline Oligomenorrhea & 74 & 5 & 79 & \\
\hline Regular & 14 & 1 & 15 & \\
\hline Hirsutism & & & & 0.901 \\
\hline Positive & 78 & 5 & 83 & \\
\hline Negative & 21 & 2 & 23 & \\
\hline \multicolumn{5}{|l|}{ LH/FSH ratio } \\
\hline$<2$ & 87 & 7 & 94 & \\
\hline$\geq 2$ & 12 & & 12 & \\
\hline Testosterone level & & & & 0.783 \\
\hline High & 7 & 1 & 8 & \\
\hline Normal & 92 & 6 & 98 & \\
\hline
\end{tabular}

results were also not significant statistically (Table 7).

\section{DISCUSSION}

The prevalence of PCOS in this study population was $33 \%$. In Pakistan, Haq et al. found that frequency of PCOS in women attending infertility clinics was $17.6 \%$ [5]. Couzin estimated that $40 \%$ of women who attend infertility clinics have PCOS. There are distinct national and ethnic differences in the incidence and presentation of PCOS: thus this syndrome varies either in its prevalence or presentation around the world or in different ethnic groups within a country [5]. The highest reported prevalence of PCOS was 52\%, among south Asian immigrants in Britain [16].

Prevalence of PCOS among infertile Kurdish women, attending infertility Care and IVF Center in Erbil was $33 \%$. In Pakistan Haq et al. found that frequency of PCOS in the infertility clinics was $17.6 \%$ [5], Couzin and Jennifer estimated that $40 \%$ of women who come to infertility clinics have PCOS.

There are national and racial differences in expression of PCOS i.e. this syndrome vary either in its prevalence or presentation around the world or in different racial groups within a country [5].

The results and percentages of the four phenotypes of women with PCOS in our study were in agreement with two studies done by Azziz [14] and Dewailly et al. [15], who also found that highest percentages of women exhibited phenotype A, having all three features of PCOS including menstrual disturbance, hirsutism, and PCO features on ultrasound scans. The least common type is phenotype $\mathrm{D}$, including women with PCOS having only hir- sutism, and PCO features on ultrasound scans.

Age is the single most important determining factor affecting female fertility, Monga [17] and Pedersen et al. [18] found that delaying marriage until after the 30s will affect the fertility rate, because fertility declines rapidly after 35 years of age. PCOS is reported to be more prevalent in younger $(<35$ years) than older women. This possibly arises from a physiological decline in the follicular cohort leading to a normalized ovarian ultrasonic appearance with advancing age [19]. The results in our study were consistent with Alnakash and Al-Taee as they noted that $87.8 \%$ of the women with PCOS included in their study were less than 35 years of age [20].

In this study, a family history of subfertility and both subfertility and obesity in mothers and sisters of women with PCOS was slightly higher than in the non-PCOS group. These results agreed with a study conducted in Alabama, USA by Kahsar et al. [21], who found the rates of PCOS in mothers and sisters of patients with PCOS were $24 \%$ and $32 \%$ respectively, although the risk was higher when considering untreated perimenopausal women only (i.e., $35 \%$ of mothers and $40 \%$ of sisters). A family history of obesity alone was not significantly higher than in the non-PCOS control group.

The presence of a high frequency of oligomenorrhea and amenorrhea among the PCOS group in the current study was in consistent with the results in a large series of women diagnosed to have PCOS by Azziz et al. [22], as approximately $75 \%-85 \%$ of women with PCOS had clinical evidence of menstrual dysfunction. Women with PCOS showed hirsutism more than women without PCOS. The prevalence and degree of hirsutism depends 
on the ethnicity of the patients. Compared with Asian women, Middle Eastern or Mediterranean women are more likely to complain of hirsutism [23]. Hirsutism is less prevalent in women with PCOS of East Asian extraction or Pacific Islanders [24] but is more prevalent in women of Indian origin [25].

Acne was more prevalent among women with PCOS than among women without PCOS. Azziz et al. [22] reported an incidence of $12 \%-14 \%$ [22], while Belosi et al. [26] reported a rate of $48.7 \%$ among women with PCOS with acne [26]; our result was between those two results.

The high rate of obesity and central obesity among infertile women in the current study can be attributed to the dietary habits (high carbohydrate/low protein diet) and the lack of exercise among Iraqi women. Elting et al. [27] also found that $11.3 \%$ of women with PCOS were overweight and $32.7 \%$ were obese. However, there are no controlled systematic studies to determine the exact prevalence of obesity in women with PCOS. Most investigators have found that $30 \%-50 \%$ of women with PCOS are obese with a tendency to have an increased WHR, or abdominal obesity [28]. There is also evidence showing that even normal weight PCOS subjects have increased intraabdominal fat [29].

In the current study, abnormally elevated LH levels and $\mathrm{LH} / \mathrm{FSH}$ ratios were found only in small percentage of women with PCOS. The characteristic increase in LH relative to FSH release has long been appreciated as a feature of PCOS. Because of the pulsatile nature of their release, a single test fails to detect an increased LH/FSH ratio. This, as well as its lack of specificity, has led to the recommendation that LH/FSH ratios should not be included in the diagnostic criteria for PCOS [20]. Thus, Cho et al. [30] found that the LH/FSH ratio had little use in diagnosing PCOS because the median LH/FSH ratio did not differ significantly between the PCOS and nonaffected group [30].

The total testosterone level was high in only $7.5 \%$ of these women with PCOS. This outcome was underestimated because we measured only total testosterone level, because of the lack of facilities for measuring free testosterone and sex hormone binding globulin in our hospital although Alnakash and Al-Taee [20] found that not all women with PCOS possess hormonal and biochemical changes suggestive of the disease.

This was consistent with the findings in the current study.

A low progesterone level (confirming failure of ovulation) in PCOS group was consistent with the findings by Sheikhha et al. [31], who found that the prevalence of infertility caused mainly by anovulation in women with PCOS varied between $35 \%$ and $94 \%$.

The high prevalence of transvaginal ultrasound fea- tures of PCO in the PCOS group was in agreement with Najem et al. [16] who reported that $74 \%$ of women with PCOS had ultrasound features of polycystic ovaries. PCO features were also found in the non-PCOS group, Balen and Michelmore found that PCO features can be detected in $19 \%$ - 33\% of the general population [16]. The low prevalence of midluteal ultrasound detection of mature follicles in the PCOS group was in agreement with Sheikhha et al. [31] who reported anovulation in $35 \%-94 \%$ of women with PCOS [32].

The traditional belief that obesity plays a serious role in the pathophysiology of PCOS dates back to the years of Stein-Leventhal but the puzzling fact is that not all women with PCOS are obese. The diversity in the criteria for diagnosing PCOS prompted us to estimate the correlations between disease manifestations and to determine whether higher BMI and WHR necessarily indicate a greater incidence of menstrual disturbance, hirsutism or higher LH/FSH ratio and elevated serum testosterone level. In the current study, the correlations between BMI and WHR and menstrual disturbances, hirsutism, LH/FSH ratio and serum testosterone level were in agreement with the findings of Alnakash and Al-Taee [20]. These findings may provide an answer to the question rose above, disproving the traditional concept of the disease that the heavier the patient, the worse is the disease manifestation.

\section{ACKNOWLEDGEMENTS}

The authors would like to thank all the women who participated in this study for their understanding of the research and giving their verbal consent to be involved in this study. The authors would like to thank the editorial services of Edanz Group Ltd. for their generous help regarding final editing of the manuscript.

\section{REFERENCES}

[1] Kauffman, R.P., Bake, T.E., Baker, V.M., DiMarino, P. and Castracane, V.D. (2008) Endocrine and metabolic differences among phenotypic expressions of polycystic ovary syndrome according to the 2003 Rotterdam consensus criteria. American Journal of Obstetrics \& Gynecology, 198, 670.e1-670.e10.

[2] Legro, R.S. (2003) polycystic ovary syndrome and cardiovascular disease: A premature association? Endocrine Reviews, 24, 302-312. doi:10.1210/er.2003-0004

[3] Balen, A. (2004) The current understanding of polycystic ovary syndrome. The Obstetrician \& Gynaecologist, 6, 66-74. doi:10.1576/toag.6.2.66.26980

[4] Kavacs, G.T. and Norman, R. (2007) Introduction: Polycystic ovary syndrome is an intergeneration problem. 2nd Edition, Monash University, Victoria.

[5] Fauzia, H., Omar, A. and Javid, R. (2007) Clinical, biochemical and ultrasonographic features of infertile women with polycystic ovarian syndrome. JCPSP, 17, 76- 
80 .

[6] Aziz, R., Woods, K.S., Reyna, R., Key, T.J., Knochenhauer, E.S. and Yildiz, B.O. (2004) The prevalence and features of the polycystic ovary syndrome in an unselected population. The Journal of Clinical Endocrinology \& Metabolism, 89, 2745-2749.

doi:10.1210/jc.2003-032046

[7] Joyce, K. (2007) Polycystic ovarian syndrome. Journal of Midwifery \& Women's Health, 51, 415-422.

[8] The Rotterdam ESHRE/ASRM Sponsored PCOS Consensus Workshop Group (2004) Revised 2003 consensus on diagnostic criteria and long-term health risks related to polycystic ovary syndrome (PCOS). Human Reproduction, 19, 41-47. doi:10.1093/humrep/deh098

[9] Abulenien, M., Barghash, A. and Mohamed, S. (2004) Clinical, ultrasonographic and endocrine predictors of ovarian response to clomiphene citrate in normogonadotropic anovulatory infertility. Middle East Fertility Society Journal, 9, 242-250.

[10] Goodman, N., Bledsoe, M., Cobin, R., Futterweit, W., Goldzieher, J., Petak, S., Smith, K. and Steinberger, E. (2001) American Association of Clinical Endocrinologists medical guidelines for the clinical practice for the diagnosis and treatment of hyperandrogenic disorders. Endocrine Practice, 7, 120-134.

[11] Ricardo, A. (2004) PCOS: A diagnostic challenge. Reproductive BioMedicine Online, 8, 644-648. doi:10.1016/S1472-6483(10)61644-6

[12] Balen, A.H., Dresner, M., Scott, E.M. and Drife, J.O. (2006) Should obese women with polycystic ovary syndrome receive treatment for infertility? British Medical Journal, 332, 434-435. doi:10.1136/bmj.332.7539.434

[13] Balen, A.H., Laven, J.S., Tan, S.L. and Dewailly, D. (2003) Ultrasound assessment of the polycystic ovary: International consensus definitions. Human Reproduction, 9, 505-514.

[14] Ricardo, A. (2006) The Rotterdam 2003 criteria for defining PCOS: CON (or how define a syndrome). The Journal of Clinical Endocrinology \& Metabolism, 17, 2005-2153.

[15] Dewailly, D., Sophie, C.-J., Anne-Celine, R., Maryse, L. and Pascal, P. (2006) Oligoovulation with polycystic ovaries but not overt hyperandrogenism. The Journal of Clinical Endocrinology \& Metabolism, 91, 3922-3927.

[16] Balen, A and Michelmore, K. (2002) what is polycystic ovary syndrome? Human Reproduction, 17, 2219-2227. doi:10.1093/humrep/17.9.2219

[17] Ash, M. (2006) Gynaecology by ten teachers. Eighteenth Edition, Arnold Holder, London, 76-88.

[18] Pedersen Sue, D., Sony, B., Peter, F. and Bernard, C. (2007) polycystic ovary syndrome. Canadian Family Physician, 53, 1041-1047.

[19] Ritta, K. (2001) Endocrine and metabolic changes in women with polycystic ovaries and polycystic ovary syndrome. University of Oulu, Oulu, 2001.

[20] Al-Nakash Abdulrazak, H. and Al-Taee Nada, K. (2007) Polycystic ovarian syndrome: The correlation between $\mathrm{LH} / \mathrm{FSH}$ ratio and disease manifestations. Middle East
Fertility Society Journal, 12, 35-40.

[21] Kahsar, M., Nixon, C., Boots, L.R., Go, R.C. and Azziz, R. (2001) Prevalence of polycystic ovary syndrome (PCOS) in first degree relatives of patients with PCOS. Fertility and Sterility, 75, 53-58. doi:10.1016/S0015-0282(00)01662-9

[22] Azziz, R., Marin, C., Hog, L., Badamgarav, E. and Song, P. (2005) Health care-related economic burden of the polycystic ovary syndrome during the reproductive life span. The Journal of Clinical Endocrinology \& Metabolism, 90, 4650-4658.

[23] Marjan, A. (2005) Polycystic ovary syndrome. Jarret Fertility Group. http://www.jarrettfertility.com/pcds.html

[24] Williamson, K., Gunn, A.J., Johnson, N. and Milsom, S.R. (2001) The impact of ethnicity on the presentation of polycystic ovarian syndrome. Australian and New Zealand Journal of Obstetrics and Gynaecology, 41, 202-206. doi:10.1111/j.1479-828X.2001.tb01210.X

[25] Wijeyaratne, C.N., Balen, A.H., Barth, J.H., Belchetz, P.E. (2002) Clinical manifestations and insulin resistance (IR) in polycystic ovary syndrome (PCOS) among South Asians and Caucasians: Is there a difference? Clinical Endocrinology (Oxford), 57, 343-350. doi:10.1046/j.1365-2265.2002.01603.x

[26] Belosi, C., Selvaggi, L., Apa, R., Guido, M., Romualdi, D., Fulghesu, A.M and Lanzone, A. (2006) Is the PCOS diagnosis solved by ESHRE/ASRM 2003 consensus or could it include ultrasound examination of the ovarian stroma? Human Reproduction, 21, 3108-3115. doi:10.1093/humrep/del306

[27] Elting, M.W., Korsen, T.J.M., Bezemer, P.D. and Schoemaker, J. (2001) Prevalence of diabetes mellitus, hypertension and cardiac complaints in q follow up study of a Dutch PCOS population. Human Reproduction, 16, 556560

[28] Wood Jennifer, R., Nelson Valen, L., Clement, H., Erik, J., Wang Clare, Y., Margrit, U., et al. (2003) The molecular phenotype of polycystic ovary syndrome (PCOS) theca cells and new candidate PCOS genes defined by microarray analysis. The Journal of Biological Chemistry, 278, 26380-26390. doi:10.1074/jbc.M300688200

[29] Yildirim, B., Sabir, N. and Kaleli, B. (2003) Relation of intra-abdominal fat distribution to metabolic disorders in non obese patients with polycystic ovary syndrome. Fertility and Sterility, 79, 1358-1364. doi:10.1016/S0015-0282(03)00265-6

[30] Cho, L.W., Jayagopal, V., Kilpatric, E.S., Holding, S. and Atkin, S.L. (2006) The LH/FSH ratio has little use in diagnosis of polycystic ovarian syndrome. Annals of Clinical Biochemistry, 43, 217-219. doi:10.1258/000456306776865188

[31] Hasan, S.M., Mehdi, K.S. and Nasrin, G. (2007) Genetics of polycystic ovary syndrome. Iranian Journal of Reproductive Medicine, 5, 1-5.

[32] Najem, F.L., Elmehdawi, R.R. and Swalem, A.M. (2007) Clinical and biochemical characteristics of polycystic ovary syndrome in Benghazi-Libya: A retrospective study. Libyan Journal of Medicine, 3, 71-74. 